Pacific Journal of Mathematics

ON THE EXISTENCE OF STRONG LIFTING IN SECOND 


\title{
ON THE EXISTENCE OF STRONG LIFTINGS IN SECOND COUNTABLE TOPOLOGICAL SPACES
}

\author{
SiEGFRIEd GRAF
}

Let $X$ be a second countable topological space, $\mathfrak{A}$ a $\sigma$-field of subsets of $X$ containing all open sets and $\mu$ a finite positive measure on $\mathfrak{A}$, such that $(X, \mathfrak{U}, \mu)$ is a complete measure space and $\mu(U)>0$ for every nonempty open $U \subset X$.

Then there exists a lifting $\phi: \mathfrak{A} \rightarrow \mathfrak{A}$ which satisfies $U \subset \phi(U)$ for every open subset $U \subset X$.

Basic notations and definitions. Throughout this paper $\mathbf{N}$ denotes the nonnegative integers and $\mathbf{R}_{+}$the nonnegative real numbers. Moreover

$X$ is a second countable topological space $(\neq \varnothing)$,

$\mathfrak{A}$ is a $\sigma$-field of subsets of $X$ containing all open sets,

$\mu: \mathfrak{Q} \rightarrow \mathbf{R}_{+}$is a countable additive measure, satisfying $\mu(U)>0$ for every nonempty open subset $U \subset X$.

For $A, B \in \mathfrak{A}$ we denote by $A \subseteq B$ the fact that $\mu(A \backslash B)=0$ and write $A \sim B$ if $A \subseteq B$ and $B \subseteq A$.

A subset $\mathscr{F} \subset \mathfrak{U}$ is called an $\cap$-system iff $\varnothing \in \mathscr{F}, X \in \mathscr{F}$ and $A \cap B \in \mathscr{F}$ for all $A, B \in \mathscr{F}$.

For an $\cap$-system $\mathscr{F} \subset \mathfrak{U}$ a mapping $\delta: \mathscr{F} \rightarrow \mathfrak{U}$ is a partial (lower) density iff it satisfies the following conditions:

(i) $A \sim B$ implies $\delta(A)=\delta(B)$

(ii) $A \sim \delta(A)$

(iii) $\delta(\varnothing)=\varnothing$ and $\delta(X)=X$

(iv) $\delta(A \cap B)=\delta(A) \cap \delta(B)$ for every $A, B \in \mathscr{F}$.

A mapping $\delta: \mathfrak{U} \rightarrow \mathfrak{U}$ with the above properties is called a (lower) density and if moreover

(v) $\delta(X \backslash A)=X \backslash \delta(A)$ for every $A \in \mathfrak{A}$ is fulfilled $\delta$ is a lifting.

A lifting or density $\delta: \mathfrak{A} \rightarrow \mathfrak{U}$ is called strong iff in addition

(vi) $U \subset \delta(U)$ for every open $U \subset X$.

Let us restate one of the fundamental properties of partial densities. For an $\cap$-system $\mathscr{F} \subset \mathfrak{A}$, a partial density $\delta: \mathscr{F} \rightarrow \mathfrak{A}$, and $A$, $B \in \mathscr{F}$ with $A \subseteq B$ we have $\delta(A) \subset \delta(B)$. In particular $\delta$ preserves inclusions. 
Existence of Strong Densities. The following two lemmas will provide us with the basic tools for the proof of the main theorem.

Lemma 1. Let $\mathscr{F} \subset \mathfrak{A}$ be an $\cap$-system, $\delta: \mathscr{F} \rightarrow \mathfrak{A}$ a partial density, and $F_{0} \in \mathfrak{U}$ arbitrary. Suppose $A_{0} \in \mathfrak{U}$ is such that $A_{0} \sim F_{0}$ and for $F^{\prime \prime}$, $F^{\prime}, F$ in $\mathscr{F}$,

$$
F^{\prime \prime} \subseteq F_{0} \text { implies } \delta\left(F^{\prime \prime}\right) \subset A_{0}
$$

and

$$
F_{0} \cap F \subseteq F^{\prime} \text { implies } A_{0} \cap \delta(F) \subset \delta\left(F^{\prime}\right) .
$$

Then $\mathscr{F}_{0}=\mathscr{F} \cup\left\{F \cap F_{0}: F \in \mathscr{F}\right\}$ is an $\cap$-system containing $\mathscr{F} \cup\left\{F_{0}\right\}$ and the mapping $\delta_{0}: \mathscr{F}_{0} \rightarrow \mathfrak{U}$ defined by

$$
\delta_{0}(F)=\left\{\begin{array}{r}
\delta(F), \quad \text { if } \quad F \in \mathscr{F} \\
\delta\left(F^{\prime}\right) \cap A_{o} \quad \text { if } \quad F=F^{\prime} \cap F_{0} \text { with } F^{\prime} \in \mathscr{F}
\end{array}\right.
$$

is a partial density which extends $\delta$.

Proof. It is obvious that $\mathscr{F}_{0}$ is an $\cap$-system containing $\mathscr{F} \cup\left\{F_{0}\right\}$. To show that $\delta_{0}$ is well-defined let $F, F^{\prime}$ be elements of $\mathscr{F}$.

(1) Assume $F \sim F^{\prime} \cap F_{0}$. This implies

$$
F \subseteq F_{0}, \quad F \subseteq F^{\prime} \text { and } F^{\prime} \cap F_{0} \subseteq F .
$$

From our assumptions concerning $A_{0}$ we may therefore conclude

$$
\delta(F) \subset A_{0} \text { and } A_{0} \cap \delta\left(F^{\prime}\right) \subset \delta(F) .
$$

Since $\delta$ is a partial density $F \subseteq F^{\prime}$ implies $\delta(F) \subset \delta\left(F^{\prime}\right)$. This inclusion combined with (*) gives us $\delta(F) \subset A_{0} \cap \delta\left(F^{\prime}\right) \subset \delta(F)$. Hence the equality $\delta(F)=A_{0} \cap \delta\left(F^{\prime}\right)$ is established.

(2) Assume $F \cap F_{0} \sim F^{\prime} \cap F_{0}$. Then we have

$$
F \cap F_{0} \subseteq F^{\prime} \text { and } F^{\prime} \cap F_{0} \subseteq F .
$$

Again it follows from our assumptions concerning $A_{0}$ that

$$
A_{0} \cap \delta(F) \subset \delta\left(F^{\prime}\right) \text { and } A_{0} \cap \delta\left(F^{\prime}\right) \subset \delta(F)
$$

and hence that

$$
A_{0} \cap \delta\left(F^{\prime}\right)=A_{0} \cap \delta(F)
$$


(1) and (2) together prove that $\delta_{0}$ is well-defined and that for $F, G \in \mathscr{F}_{0}$ the fact $F \sim G$ implies $\delta_{0}(F)=\delta_{0}(G)$. According to its definition $\delta_{0}$ obviously satisfies the other conditions for a partial density. It is also clear that $\delta_{0}$ extends $\delta$.

Lemma 2. Let $\mathscr{F} \subset \mathfrak{A}$ be a countable $\cap$-system, $F_{0} \in \mathfrak{A}$ arbitrary, $\mathscr{F}_{0}=\mathscr{F} \cup\left\{F \cap F_{0}: F \in \mathscr{F}\right\}$ and $\delta: \mathscr{F} \rightarrow \mathfrak{A}$ a partial density. Then there exists a partial density $\delta_{0}: \mathscr{F}_{0} \rightarrow \mathfrak{U}$ which extends $\delta$.

Proof. According to Lemma 1 the assertion of Lemma 2 is true if we can prove the existence of a set $A_{0} \in \mathfrak{U}$ such that $F_{0} \sim A_{0}$ and for $F$, $F^{\prime}, F^{\prime \prime}$ in $\mathscr{F}$,

$$
F^{\prime \prime} \subseteq F_{0} \text { implies } \delta\left(F^{\prime \prime}\right) \subset A_{0}
$$

and

$$
F_{0} \cap F \subset F^{\prime} \text { implies } A_{0} \cap \delta(F) \subset \delta\left(F^{\prime}\right) \text {. }
$$

As an easy calculation shows $A_{0} \in \mathfrak{A}$ fulfills the last two conditions if and only if

$$
\delta\left(F^{\prime \prime}\right) \subset A_{0} \subset \delta\left(F^{\prime}\right) \cup C \delta(F)
$$

whenever $F, F^{\prime}, F^{\prime \prime} \in \mathscr{F}$ satisfy $F^{\prime \prime} \subseteq F_{0} \subseteq F^{\prime} \cup C F$.

To establish the existence of such an $A_{0}$ let

$$
\mathscr{G}:=\left\{\delta\left(F^{\prime \prime}\right): F^{\prime \prime} \subset \mathscr{F} \text { and } F^{\prime \prime} \subseteq F_{0}\right\}
$$

and

$$
\mathscr{H}:=\left\{\delta\left(F^{\prime}\right) \cup \boldsymbol{C} \delta(F): F, F^{\prime} \in \mathscr{F} \text { and } F_{0} \subseteq F^{\prime} \cup C F\right\}
$$

Since $\mathscr{F}$ is countable the sets $\mathscr{G}$ and $\mathscr{H}$ are also countable.

Therefore $A:=\cup \mathscr{G}$ and $B:=\cap \mathscr{H}$ are in $\mathfrak{A}$.

Property (ii) of partial densities implies

$$
G \subseteq F_{0} \text { and } F_{0} \subseteq H
$$

for every $G \in \mathscr{G}$ and $H \in \mathscr{H}$. Due to the countable additivity of $\mu$ we therefore get:

$$
A \subseteq F_{0} \text { and } F_{0} \subseteq B
$$

Let $G \in \mathscr{G}$ and $H \in \mathscr{H}$ be arbitrary. Then there are $F, F^{\prime}, F^{\prime \prime} \in \mathscr{F}$ with $F^{\prime \prime} \subseteq F_{0} \subseteq F^{\prime} \cup C F, G=\delta\left(F^{\prime \prime}\right)$, and $H=\delta\left(F^{\prime}\right) \cup C \delta(F)$. From $F^{\prime \prime} \subseteq$ $F^{\prime} \cup C F$ we conclude 


$$
F^{\prime \prime} \cap F \subseteq F^{\prime} \text { and hence } \delta\left(F^{\prime \prime}\right) \cap \delta(F) \subset \delta\left(F^{\prime}\right)
$$

which implies $G=\delta\left(F^{\prime \prime}\right) \subset \delta\left(F^{\prime}\right) \cup C \delta(F)=H$. This gives us

$$
A \subset B .
$$

It is a consequence of $(\dagger)$ and ( $\ddagger)$ that

$$
A_{0}:=\left(F_{0} \cup A\right) \cap B \sim F_{0}
$$

and

$$
G \subset A_{0} \subset H
$$

for all $G \in \mathscr{G}, H \in \mathscr{H}$.

Hence the statement of Lemma 2 is proved.

PROPOSITION 1. Let $\mathscr{B}$ be a countable base of the topology of $X$ and $\mathscr{F}$ the field generated by $\mathscr{B}$. Then there is a partial density $\delta: \mathscr{F} \rightarrow \mathfrak{A}$ satisfying $U \subset \delta(U)$ for every $U \in \mathscr{B}$.

Proof. Without loss of generality we may assume that $\mathscr{B}$ is stable under finite unions and intersections and contains $\varnothing$ and $X$.

For $B \in \mathscr{B}$ let $\mathscr{B}(B):=\{A \in \mathscr{B}: A \sim B\}$ and define $\phi: \mathscr{B} \rightarrow \mathfrak{A}$ by $\phi(B)=\cup \mathscr{B}(B)$. Since $\cup \mathscr{B}(B)$ is open for every $B \in \mathscr{B}$ and since $\mathfrak{A}$ contains all open sets $\phi$ is a well-defined map from $\mathscr{B}$ to $\mathfrak{A}$. It is an immediate consequence of the definition of $\phi$ that for $A, B \in \mathscr{B}$ the equivalence $A \sim B$ implies $\phi(A)=\phi(B)$. Since $\mathscr{B}(B)$ is countable we have $B \sim \phi(B)$.

According to our assumptions every nonempty open set has strictly positive measure. Therefore $\mathscr{B}(\varnothing)=\{\varnothing\}$ and hence $\phi(\varnothing)=\varnothing$.

Since $X \in \mathscr{B}(X)$ the condition $\phi(X)=X$ is fulfilled.

$\phi$ also preserves finite intersections. To show this let $A, B \in \mathscr{B}$ be arbitrary. Because $\mathscr{B}$ is stable under finite intersections we have

$$
\left\{A^{\prime} \cap B^{\prime}: A^{\prime} \in \mathscr{B}(A), B^{\prime} \in \mathscr{B}(B)\right\} \subset \mathscr{B}(A \cap B)
$$

and consequently

$$
\begin{aligned}
\phi(A) \cap \phi(B) & =(\cup \mathscr{B}(A)) \cap(\cup \mathscr{B}(B)) \\
& =\cup\left\{A^{\prime} \cap B^{\prime}: A^{\prime} \in \mathscr{B}(A), B^{\prime} \in \mathscr{B}(B)\right\} \\
& \subset \cup \mathscr{B}(A \cap B)=\phi(A \cap B) .
\end{aligned}
$$

To prove the inverse inclusion let $C \in \mathscr{B}(A \cap B)$ be arbitrary. Since $\mathscr{B}$ is stable under finite unions we have $A \cup C \in \mathscr{B}(A)$ and hence $C \subset \phi(A)$. For the same reasons $C \subset \phi(B)$ is true and therefore the inclusion 


$$
\phi(A \cap B) \subset \phi(A) \cap \phi(B)
$$

holds.

Using Lemma 2 , the fact that $\mathscr{F}$ is countable, and the fact that $\mathscr{B}$ is an $\cap$-system contained in $\mathscr{F}$, we see by induction that $\phi$ can be extended to a partial density $\delta: \mathscr{F} \rightarrow \mathfrak{A}$. From the definition of $\phi$ it follows immediately that $U \subset \phi(U)=\delta(U)$ for all $U \in \mathscr{B}$.

The following two propositions are stated without proofs. The proofs can be found in [4], although a slightly different notation is used there.

Proposition 2. ([4], p. 64, Lemma 4.6). Let $\left(\mathfrak{A}_{n}\right)_{n \in \mathrm{N}}$ be a sequence of $\sigma$-fields of subsets of $X$ with

$$
\mathfrak{A}_{\mathbf{n}} \subset \mathfrak{U}_{\mathrm{n}+1} \subset \mathfrak{U}
$$

and $\delta_{n}: \mathfrak{A}_{n} \rightarrow \mathfrak{A}$ a partial density with

$$
\delta_{n+1} \mid \mathfrak{U}_{n}=\delta_{n}
$$

for every $n \in \mathbf{N}$.

Denote by $\mathfrak{A}_{\infty}$ the $\sigma$-field generated by $\cup\left\{\mathfrak{A}_{n}: n \in \mathbf{N}\right\}$. Then there exists a partial density $\delta_{\infty}: \mathfrak{A}_{\infty} \rightarrow \mathfrak{U}$ satisfying $\delta_{\infty} \mid \mathfrak{A}_{n}=\delta_{n}$ for all $n \in \mathbf{N}$.

Though the above proposition is not explicity stated in his paper Traynor proved it independently in [8].

Proposition 3. ([4], p. 61, Satz. 4.3 and Lemma 4.4). Let $\mathscr{L}$ be a $\sigma$-field, $A_{0} \in \mathfrak{U}$ arbitrary, and $\delta: \mathscr{L} \rightarrow \mathfrak{A}$ a partial density.

Then $\mathscr{L}_{0}:=\left\{\left(B \cap A_{0}\right) \cup\left(B^{\prime} \backslash A_{0}\right): B, B^{\prime} \in \mathscr{L}\right\}$ is a $\sigma$-field containing $\mathscr{L} \cup\left\{A_{0}\right\}$ and there exists a partial density $\delta_{0}: \mathscr{L}_{0} \rightarrow \mathfrak{A}$ extending $\delta . \quad \delta_{0}$ can be defined by

$$
\begin{aligned}
& \delta_{0}\left(\left(B \cap A_{0}\right) \cup\left(B^{\prime} \backslash A_{0}\right)\right) \\
= & \left(\delta\left(\left(B \cap B_{1}\right) \cup\left(B^{\prime} \backslash B_{1}\right)\right) \cap A_{0}\right) \cup\left(\delta\left(\left(B \backslash B_{2}\right) \cup\left(B^{\prime} \cap B_{2}\right)\right) \backslash A_{0}\right),
\end{aligned}
$$

where $B, B^{\prime}$ are elements of $\mathscr{L}$ and where $B_{1} \in \mathscr{L}$ satisfies $A_{0} \subseteq B_{1}$ and $B_{1} \subseteq A$ for every $A \in \mathscr{L}$ with $A_{0} \subseteq A$ while $B_{2} \in \mathscr{L}$ satisfies $C A_{0} \subseteq B_{2}$ and $B_{2} \subseteq C$ for every $C \in \mathscr{L}$ with $C \widetilde{A}_{0} \subseteq C$.

THEOREM 1. There exists a strong lower density $\rho: \mathfrak{A} \rightarrow \mathfrak{A}$.

Proof. Let $\mathscr{B}$ be a countable base for the topology of $X$ and $\mathscr{F}$ the field generated by $\mathscr{B}$. According to Proposition 1 there is a partial density $\delta: \mathscr{F} \rightarrow \mathfrak{A}$ such that $U \subset \delta(U)$ for every $U \in \mathscr{B}$. 
Let $f: \mathbf{N} \rightarrow \mathscr{F}$ be a bijection and $\mathfrak{U}_{n}$ the field generated by $\{f(m): 0 \leqq$ $m \leqq n\}$. It is clear that $\mathfrak{A}_{n} \subset \mathscr{F}$ is a $\sigma$-field. Define $\delta_{n}: \mathfrak{A}_{n} \rightarrow \mathfrak{A}$ by $\delta_{n}=\delta \mid \mathfrak{A}_{n}$. Then $\left(\mathfrak{A}_{n}, \delta_{n}\right)_{n \in \mathbb{N}}$ fulfills the assumptions of Proposition 2. Thus there is a partial density $\delta_{\infty}: \mathfrak{A}_{\infty} \rightarrow \mathfrak{U}_{\infty}$ extending $\delta$, where $\mathfrak{A}_{\infty}$ is the $\sigma$-field generated by $\mathscr{F}=\cup\left\{\mathfrak{A}_{n}: n \in \mathbf{N}\right\}$. Using Zorn's lemma and Propositions 2 and 3 we will show that $\delta_{\infty}$ can be extended to a density $\rho: \mathfrak{U} \rightarrow \mathfrak{A}$.

To this purpose define

$$
\begin{gathered}
\mathscr{T}:=\left\{(\mathscr{L}, \psi): \mathscr{L} \boldsymbol{\sigma} \text {-field containing } \mathfrak{A}_{\infty} \text { and } \psi: \mathscr{L} \rightarrow \mathfrak{A}\right. \\
\text { a partial density with } \left.\psi \mid \mathfrak{A}_{\infty}=\delta_{\infty}\right\} .
\end{gathered}
$$

For $(\mathscr{L}, \psi),\left(\mathscr{L}^{\prime}, \psi^{\prime}\right) \in \mathscr{T}$ let $(\mathscr{L}, \psi) \leqq\left(\mathscr{L}^{\prime}, \psi^{\prime}\right)$ denote the fact that $\mathscr{L} \subset \mathscr{L}^{\prime}$ and $\psi^{\prime} \mid \mathscr{L}=\psi$. It is easy to check that $\leqq$ is an order-relation on $\mathscr{T}$.

Next we will prove that $\mathscr{T}$ is inductively orderby by $\leqq$. Let $\mathscr{K} \subset \mathscr{L}$ be any totally ordered subset. The following two cases have to be considered.

(1) If for every sequence $\left(\mathscr{L}_{n}, \psi_{n}\right)_{n \in N}$ in $\mathscr{K}$ there is a $(\mathscr{L}, \psi) \in \mathscr{K}$ with $\left(\mathscr{L}_{n}, \psi_{n}\right) \leqq(\mathscr{L}, \psi)$ for all $n \in \mathbf{N}$, then $\overline{\mathscr{L}}:=\cup\{\mathscr{L}:(\mathscr{L}, \psi) \in \mathscr{K}\}$ is a $\sigma$-field containing $\mathfrak{A}_{\infty}$ and $\bar{\psi}: \overline{\mathscr{L}} \rightarrow \mathfrak{U}$ defined by $\bar{\psi} \mid \mathscr{L}=\psi$ for every $(\mathscr{L}, \psi) \in \mathscr{K}$ is a partial density extending $\delta_{\infty}$. Hence we have $(\overline{\mathscr{L}}, \bar{\psi}) \in \mathscr{T}$ and $(\mathscr{L}, \psi) \leqq(\mathscr{L}, \bar{\psi})$ for all $(\mathscr{L}, \psi) \in \mathscr{K}$.

(2) If there exists a sequence $\left(\mathscr{L}_{n}, \psi_{n}\right)_{n \in N}$ in $\mathscr{K}$ such that for each $(\mathscr{L}, \psi) \in \mathscr{K}$ there is an $n \in \mathbf{N}$ with $(\mathscr{L}, \psi) \leqq\left(\mathscr{L}_{n}, \psi_{n}\right)$ we have

$$
\cup\{\mathscr{L}:(\mathscr{L}, \psi) \in \mathscr{K}\}=\cup\left\{\mathscr{L}_{n}: n \in \mathbf{N}\right\}=\overline{\mathscr{L}} .
$$

Let $\mathscr{L}_{\infty}$ denote the $\sigma$-field generated by $\mathscr{L}$. From Proposition 2 we conclude the existence of a partial density $\psi_{\infty}: \mathscr{L}_{\infty} \rightarrow \mathfrak{A}$ which extends all $\psi_{n}$ and hence we get $(\mathscr{L}, \psi) \leqq\left(\mathscr{L}_{\infty}, \psi_{\infty}\right) \in \mathscr{T}$ for all $(\mathscr{L}, \psi) \in \mathscr{K}$.

Since $\left(\mathfrak{A}_{\infty}, \delta_{\infty}\right) \in \mathscr{T}$ and thus $\mathscr{T} \neq \varnothing$, Zorn's lemma gives us the existence of a maximal element $(\mathscr{\mathscr { F }}, \rho)$ in $\mathscr{T}$.

From Proposition 3 we conclude $\overline{\mathscr{F}}=\mathfrak{A}$ and hence $\rho$ is a density. To prove the theorem it remains to check that $\rho$ is strong. To this end let $U$ be any open subset of $X$. Then

$$
U=\cup\{B \in \mathscr{B}: B \subset U\}
$$

for $B \in \mathscr{B}$ and $B \subset U$ we have

$$
\rho(U) \supset \rho(B)=\delta_{\infty}(B)=\delta(B)=\delta(B) \supset B
$$

and thus

$$
U=\cup\{B \in \mathscr{B}: B \subset U\} \subset \rho(U) .
$$

Hence $\rho$ is strong. 
The main theorem. To verify the main theorem we need one more lemma which is an immediate consequence of a more general theorem of von Neumann and Stone ([6], p. 372, Th. 18). Easier direct proofs were given by Sion in [7], Gapaillard in [3], Traynor in [8] and the author in [4]. Let us restate this lemma but omit the proof.

LEMma 3. If $(X, \mathfrak{A}, \mu)$ is complete and $\delta: \mathfrak{A} \rightarrow \mathfrak{A}$ a density then there is a lifting $\phi: \mathfrak{A} \rightarrow \mathfrak{U}$ with $\delta(A) \subset \phi(A)$ for every $A \in \mathfrak{A}$.

THEOREM 2. If $(X, \mathfrak{A}, \mu)$ is complete then there exists a strong lifting $\phi ; \mathfrak{A} \rightarrow \mathfrak{A}$.

Proof. According to Theorem 1 there is a strong density $\delta: \mathfrak{A} \rightarrow$ A. From Lemma 3 we get a lifting $\phi ; \mathfrak{A} \rightarrow \mathfrak{U}$ satisfying $\delta(A) \subset \phi(A)$ for all $A \in \mathfrak{A}$. Since $\delta$ is strong we therefore conclude $U \subset \delta(U) \subset$ $\phi(U)$ for every open set $U \subset X$.

Hence $\phi$ is a strong lifting.

Notes.

(1) All previous statements of this paper remain true for $\sigma$-finite measure spaces because for any $\sigma$-finite measure on a $\sigma$-field there exists a finite measure with the same nullsets.

(2) The methods of proofs show that for an arbitrary finite or $\sigma$-finite measure space $(Y, \mathscr{L}, \nu)$ and any countable $\mathscr{F} \subset \mathscr{L}$, such that for each finite $\mathscr{G} \subset \mathscr{F}$ with $\nu(\cap \mathscr{G})=0$ we have $\cap \mathscr{G}=\varnothing$, there is a density $\delta: \mathscr{L} \rightarrow \mathscr{L}$ satisfying $F \subset \delta(F)$ for all $F \in \mathscr{F}$.

If $(Y, \mathscr{L}, \nu)$ is furthermore complete then there is even a lifting $\phi: \mathscr{L} \rightarrow \mathscr{L}$ with these properties.

(3) Let $X$ be a second countable topological space, $\mathfrak{A}$ the Borelfield in $X$ and $\mathfrak{W}$ a $\sigma$-ideal in $\mathfrak{A}$ containing no nonempty open sets. Then $\mathfrak{A}$ has at most the power of the continuum. Assuming the continuum hypothesis and using the same methods as in the first part of this paper we get a strong density $\delta ; \mathfrak{U} \rightarrow \mathfrak{A}$ with respect to $\mathfrak{B}$. From a lifting theorem of von Neumann-Stone (certificate [6] or [4], p. 47, Satz 3.3) we may conclude (using the continuum hypothesis another time) that there is even a strong lifting $\phi: \mathfrak{A} \rightarrow \mathfrak{U}$.

(4) The existence of a strong lifting was well-known for separable metric spaces with finite outer regular measures strictly positive on non-empty open sets (see Ionescu-Tulcea [5] and Sion [7]) and for locally compact metrizable spaces with (not necessarily $\sigma$-finite) Radon measures whose support is the whole space (see Ionescu-Tulcea [5]). The first fact is a special case of the main theorem of this paper while the second fact can be derived from this theorem using Proposition 2, p. 108 in Ionescu-Tulcea [5], and observing that any metrizable compact space is separable.

(5) Bichteler proves in [1] a strong-lifting-theorem implying the existence of a strong lifting for locally compact metrizable spaces with 
Radon measures whose support is the whole space. But the result of this paper are no (immediate) consequences of Bichteler's theorem.

(6) Eifrig [2] gives a proof of the existence of a strong lifting for the interval $[0,1]$ using methods of nonstandard-analysis.

(7) For applications of strong-lifting-theorems to integral representations and disintegration of measures see Ionescu-Tulcea [5] and Sion [7].

\section{REFERENCES}

1. K. Bichteler, Integration Theory (with Special Attention to Vector Measures), Lect. Notes in Mathematics 315 (1973), Springer Verlag, Berlin-Heidelberg-New York.

2. B. Eifrig, Ein nicht-standard Beweis für die Existenz eines starken Liftings in $\mathscr{L}_{\infty}([0,1[)$, Contributions to Nonstandard Analysis, Edited by W.A.J. Luxemburg and A. Robinson, North Holland Publ. Co., Amsterdam 1972.

3. J. Gapaillard, Relevements, Bases de derivation ensemblistes et fonctionelles, These, Nantes 1972.

4. S. Graf, Schnitte Boolescher Korrespondenzen und ihre Dualisierungen, Dissertation, Erlangen 1973.

5. A. Ionescu Tulcea and C. Ionescu Tulcea, Topics in the Theory of Lifting, Springer Verlag, Berlin-Heidelberg - New York 1969.

6. J. von Neumann and M. H. Stone, The determination of representative elements in the Residual classes of a Boolean algebra, Fund. Math., 25 (1935), 353-376.

7. M. Sion, A theory of Semigroup Valued Measure, Lect. Notes in Mathematics, 355 (1973), Springer Verlag, Berlin-Heidelberg - New York.

8. T. Traynor, An Elementary Proof of the Lifting Theorem, Pacific J. Math., 53 (1974), 267-272.

Received May 13, 1974.

UNIVERSITY OF BRITISH COLUMBIA 


\section{PACIFIC JOURNAL OF MATHEMATICS}

\section{EDITORS}

RICHARD ARENS (Managing Editor)

University of California

Los Angeles, California 90024

\section{J. DugundJI}

Department of Mathematics University of Southern California Los Angeles, California 90007

D. Gilbarg and J. Milgram

Stanford University

Stanford, California 94305

\section{ASSOCIATE EDITORS}
E. F. BECKENBACH
B. H. NeumanN
F. WoLF
K. YoshiDA

\section{SUPPORTING INSTITUTIONS}

\author{
UNIVERSITY OF BRITISH COLUMBIA \\ CALIFORNIA INSTITUTE OF TECHNOLOGY \\ UNIVERSITY OF CALIFORNIA \\ MONTANA STATE UNIVERSITY \\ UNIVERSITY OF NEVADA \\ NEW MEXICO STATE UNIVERSITY \\ OREGON STATE UNIVERSITY \\ UNIVERSITY OF OREGON \\ OSAKA UNIVERSITY
}

\author{
UNIVERSITY OF SOUTHERN CALIFORNIA \\ STANFORD UNIVERSITY \\ UNIVERSITY OF TOKYO \\ UNIVERSITY OF UTAH \\ WASHINGTON STATE UNIVERSITY \\ UNIVERSITY OF WASHINGTON \\ AMERICAN MATHEMATICAL SOCIETY
}

The Supporting Institutions listed above contribute to the cost of publication of this Journal, but they are not owners or publishers and have no responsibility for its contents or policies.

Mathematical papers intended for publication in the Pacific Journal of Mathematics should be in typed form or offset-reproduced (not dittoed), double spaced with large margins. Underline Greek letters in red, German in green, and script in blue. The first paragraph or two must be capable of being used separately as a synopsis of the entire paper. Items of the bibliography should not be cited there unless absolutely necessary, in which case they must be identified by author and Journal, rather than by item number. Manuscripts, in duplicate, may be sent to any one of the four editors. Please classify according to the scheme of Math. Reviews, Index to Vol. 39. All other communications should be addressed to the managing editor, or Elaine Barth, University of California, Los Angeles, California, 90024.

100 reprints are provided free for each article, only if page charges have been substantially paid. Additional copies may be obtained at cost in multiples of 50 .

The Pacific Journal of Mathematics is issued monthly as of January 1966. Regular subscription rate: $\$ 72.00$ a year (6 Vols., 12 issues). Special rate: $\$ 36.00$ a year to individual members of supporting institutions.

Subscriptions, orders for back numbers, and changes of address should be sent to Pacific Journal of Mathematics, 103 Highland Boulevard, Berkeley, California, 94708.

PUBLISHED BY PACIFIC JOURNAL OF MATHEMATICS, A NON-PROFIT CORPORATION Printed at Jerusalem Academic Press, POB 2390, Jerusalem, Israel.

$$
\begin{gathered}
\text { Copyright (C) } 1975 \text { Pacific Journal of Mathematics } \\
\text { All Rights Reserved }
\end{gathered}
$$




\section{Pacific Journal of Mathematics}

\section{Vol. 58, No. $2 \quad$ April, 1975}

Zvi Artstein and John Allen Burns, Integration of compact set-valued functions . . . . . . . . . 297

Mark Benard, Characters and Schur indices of the unitary reflection group $[321]^{3} \ldots \ldots \ldots . .309$

Simeon M. Berman, A new characterization of characteristic functions of absolutely continuous

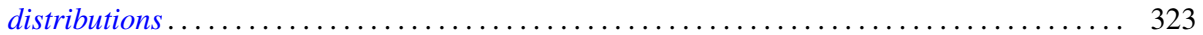

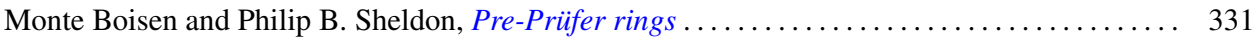

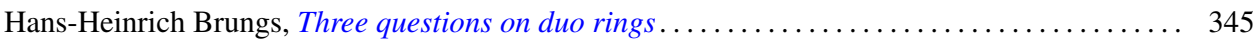

Iracema M. Bund, Birnbaum-Orlicz spaces of functions on groups................. 351

John D. Elwin and Donald R. Short, Branched immersions between 2-manifolds of higher

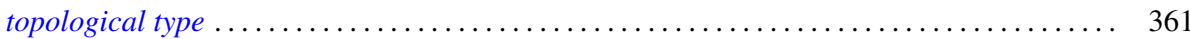

Eric Friedlander, Extension functions for rank 2, torsion free abelian groups . .......... 371

Jon Froemke and Robert Willis Quackenbush, The spectrum of an equational class of

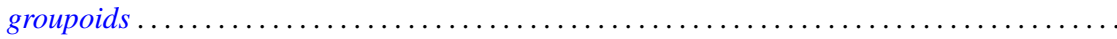

Barry J. Gardner, Radicals of supplementary semilattice sums of associative rings ...........

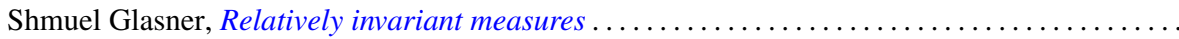

George Rudolph Gordh, Jr. and Sibe Mardesic, Characterizing local connectedness in inverse

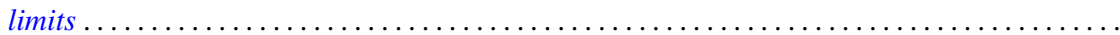

Siegfried Graf, On the existence of strong liftings in second countable topological spaces......

Stanley P. Gudder and D. Strawther, Orthogonally additive and orthogonally increasing

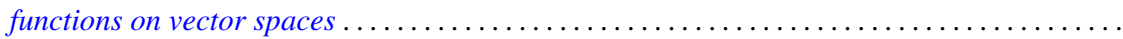

Darald Joe Hartfiel and Carlton James Maxson, A characterization of the maximal monoids and

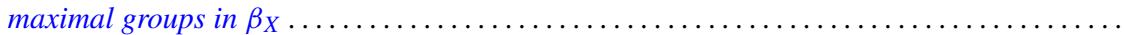

Robert E. Hartwig and S. Brent Morris, The universal flip matrix and the generalized faro-shuffle. .

William Emery Haver, Mappings between ANRs that are fine homotopy equivalences. .

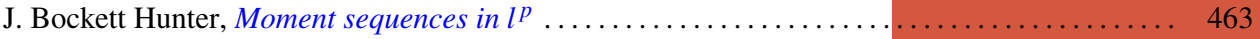

Barbara Jeffcott and William Thomas Spears, Semimodularity in the completion of a poset.... 467

Jerry Alan Johnson, A note on Banach spaces of Lipschitz functions . . . . . . . . . . . . 475

David W. Jonah and Bertram Manuel Schreiber, Transitive affine transformations on

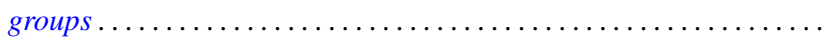

Karsten Juul, Some three-point subset properties connected with Menger's characterization of

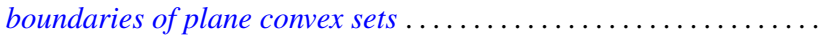

Ronald Brian Kirk, The Haar integral via non-standard analysis . . . . . . . . . . . . . 517

Justin Thomas Lloyd and William Smiley, On the group of permutations with countable

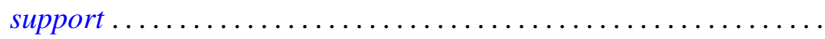

Erwin Lutwak, Dual mixed volumes .................................. 531

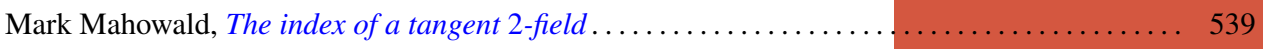

Keith Miller, Logarithmic convexity results for holomorphic semigroups . . . . . . . . . . . . 549

Paul Milnes, Extension of continuous functions on topological semigroups . . . . . . . . . . 553

Kenneth Clayton Pietz, Cauchy transforms and characteristic functions ................ 563

James Ted Rogers Jr., Whitney continua in the hyperspace $C(X) \ldots \ldots \ldots \ldots \ldots \ldots \ldots \ldots .569$

Jean-Marie G. Rolin, The inverse of a continuous additive functional . . . . . . . . . . . . 585

William Henry Ruckle, Absolutely divergent series and isomorphism of subspaces . ........ 605

Rolf Schneider, A measure of convexity for compact sets . ..................... 617

Alan Henry Schoenfeld, Continous measure-preserving maps onto Peano spaces .......... 627

V. Merriline Smith, Strongly superficial elements .......................... 643

Roger P. Ware, A note on quadratic forms over Pythagorean fields . . . . . . . . . . . . . . 651

Roger Allen Wiegand and Sylvia Wiegand, Finitely generated modules over Bezout rings . . . . 655

Martin Ziegler, A counterexample in the theory of definable automorphisms . . . . . . . . . 665 